Cite as: Janacsek, K., \& Nemeth, D. (2013). Implicit sequence learning and working memory: correlated or complicated?. Cortex, 49(8), 2001-2006.

\title{
Implicit Sequence Learning and Working Memory: Correlated or Complicated?
}

$$
\text { Janacsek K. }{ }^{1} \text { \& Nemeth, D. }{ }^{1,2}
$$

${ }^{1}$ Department of Clinical Psychology and Addiction, Eotvos Lorand University, Budapest, Hungary

${ }^{2}$ Imaging Research Center, University of Texas, Austin, USA

The authors report no conflict of interest and have no financial disclosure.

Corresponding author: Dezso Nemeth, Ph.D., Institute of Psychology, Eotvos Lorand University, Budapest, Hungary, Email: nemethd@gmail.com, WEB: www.memory-and$\underline{\text { language.com }}$ 


\begin{abstract}
The relationship between implicit/incidental sequence learning and working memory motivated a series of research because it is plausible that higher working memory capacity opens a "larger window" to a sequence, allowing thereby the sequence learning process to be easier. Although the majority of studies found no relationship between implicit sequence learning and working memory capacity, in the past few years several studies have tried to demonstrate the shared or partly shared brain networks underlying these two systems. In order to help the interpretation of these and future results, in this mini-review we suggest the following factors to be taken into consideration before testing the relationship between sequence learning and working memory: 1) the explicitness of the sequence; 2) the method of measuring working memory capacity; 3) online and offline stages of sequence learning; and 4) general skill- and sequence-specific learning.
\end{abstract}

Keywords: skill learning, sequence learning, implicit vs. explicit learning, working memory, dorsolateral prefrontal cortex 
Although implicit sequence learning is a subconscious process which is believed to be independent from general cognitive resources such as working memory, in the past few years several studies have set out to demonstrate the shared or partly shared brain networks underlying these two systems. For example, disrupting the dorsolateral prefrontal cortex (DLPFC), a structure involved in working memory, with transcranial magnetic stimulation (TMS) impairs implicit sequence learning (Pascual-Leone, Wassermann, Grafman, and Hallett, 1996; Robertson, Tormos, Maeda, and Pascual-Leone, 2001). However, the role of PFC in implicit sequence learning is controversial: while some studies found activation of the DLPFC in implicit sequence learning (Pascual-Leone et al., 1996; Robertson et al., 2001; Schwarb and Schumacher, 2009), others failed to find such a relationship (Bo, Peltier, Noll, and Seidler, 2011; Fletcher et al., 2005; Rieckmann, Fischer, and Bäckman, 2010). Moreover, several studies showed that manipulations reducing the dominance of the PFC and/or the medial temporal lobe (MTL), such as a demanding secondary task (Foerde, Knowlton, and Poldrack, 2006), a distractor task inserted between the learning sessions (R. M. Brown and Robertson, 2007), hypnosis during learning (Nemeth, Janacsek, Polner, and Kovacs, in press) or neuropharmacological blockage (Frank, O'Reilly, and Curran, 2006), had no effect or even led to performance improvements in sequence learning tasks. These latter findings support the competitive nature of the PFC- and MTL-dependent and basal ganglia-dependent memory systems (Poldrack et al., 2001).

To refine the interpretation of these and future results, we outline several factors in this mini-review to be taken into consideration before planning brain imaging, psychophysiology, and behavioral studies on the relationship between sequence learning and working memory. 


\section{Evidence for independence between implicit sequence learning and working memory}

The relationship between implicit sequence learning and working memory motivated a series of research because it is plausible to suggest that higher working memory capacity opens a "larger window" to a sequence, allowing thereby the sequence learning process to be easier (Frensch and Miner, 1994; Howard and Howard, 1997). However, the majority of studies (see Table 1) found no relationship between implicit sequence learning and working memory capacity. For instance, Feldman et al. (1995) demonstrated that there is no significant correlation between sequence learning scores (performance on a random block minus performance on a sequence block) on a 10-element deterministic implicit serial reaction time (SRT) task and span tasks (Digit and Backward Digit Span Tasks; and Wisconsin Card Sorting Test). Unsworth and Engle (2005) found that high and low working memory capacity individuals (measured by Operation Span Task) did not differ in performance on implicit sequence learning; moreover, the implicit sequence learning was independent from general fluid intelligence. Kaufman et al. (2010) found similar results using a probabilistic implicit sequence learning task and demonstrated with structural equation modeling that working memory is independent from implicit learning. Frensch and Miner (1994) also failed to find a significant correlation between implicit/incidental sequence learning in the single-task condition and performance on span tasks. Bo et al. $(2011,2012)$ did not find a correlation between classical learning score on the SRT task and working memory measures either.

Neuropsychological investigations also suggest the independence of implicit sequence learning and working memory. For example, a recent study found working memory deficits,but intact implicit sequence learning abilities in individuals with Obstructive Sleep Apnea (Nemeth, Csábi, Janacsek, Varszegi, and Mari, 2012). In addition, several studies showed intact implicit sequence learning in groups with intellectual disabilities, for example in Autistic Spectrum Disorder (Barnes et al., 2008; J. Brown, Aczel, Jimenez, Kaufman, and 
Grant, 2010; Nemeth, Janacsek, Balogh et al., 2010) or Down-Syndrome (Vicari, Verucci, and Carlesimo, 2007). As working memory is highly correlated with general intellectual abilities while implicit learning is independent of IQ, (e.g., Kaufman et al., 2010), we can interpret these results as indirect evidence for independence between implicit sequence learning and working memory. In sum, despite partly overlapping brain networks (PascualLeone et al., 1996; Sefcsik et al., 2009), these two systems seem to be separate from each other on the functional level.

\section{Factors influencing effects of working memory on sequence learning}

Explicitness of the sequence - If the sequence learning is explicit/intentional, working memory differences emerge in the sequence learning tasks (Unsworth and Engle, 2005). Frensch and Miner (1994, Experiment 1), as well as Bo and colleagues (Bo, Borza, and Seidler, 2009; Bo et al., 2012), found significant correlation between working memory and some measures of explicit sequence learning. These studies suggest that working memory is engaged in explicit learning to guide the focus of attention and cognitive control (Cowan, 1995; Jiménez, 2003; Kaufman et al., 2010). This idea is also supported by the more attention demanding dual-task experiments (Frensch and Miner, 1994, Experiment 2) in which sequence learning performance under dual-task conditions correlated with Digit Span and Location Span Tasks. In line with this argument, functional magnetic resonance imaging (fMRI) and positron emission tomography (PET) studies of sequence learning found greater activity in prefrontal cortical areas during explicit sequence learning compared to the implicit condition (Destrebecqz et al., 2005; Fletcher et al., 2005; Honda et al., 1998). Prefrontal cortical areas are thought to be engaged in working memory performance as well (Champod and Petrides, 2010; Smith and Jonides, 1999). 
Measures of working memory - As the above mentioned studies have shown, different methods could lead to different working memory effects on sequence learning. Performance on short-term and working memory span tasks (e.g., Forward and Backward Digit Span, Operation Span, Reading Span, and Listening Span Tasks) shows no correlation with implicit/incidental sequence learning, while dual-task methods (Frensch and Miner, 1994) and change detection working memory tasks (Bo et al., 2011) can demonstrate working memory effects on implicit sequence learning. In contrast, most studies have found working memory effects on explicit sequence learning using any type of WM measure (e.g., Unswoth and Engle, 2005; Bo et al., 2009; Weitz et al., 2011). In addition, we also have to consider the difference between verbal and visuospatial working memory depending on whether verbal (e.g., letters, digits, words) or visuospatial material (e.g., shapes, colors, locations) needs to be remembered. These two types of working memory can relate to sequence learning in different ways, suggesting some extent of domain-specificity. One can assume that the performance in a sequence learning task, where the sequence is defined as a stimulus-series of different locations (e.g., classical SRT task, Nissen and Bullemer, 1987), might correlate stronger with visuospatial than with verbal working memory capacity. For example, in the study of French and Miner (1994, Experiment 1), visuospatial sequence learning correlated with Location Span but not with Digit Span. Similarly, Bo et al. (2009) found a relationship between sequence learning, measured by the chunk length of a visuospatial sequence learned by the participants, and visuospatial working memory capacity. In contrast, verbal working memory might play a greater role in sequence learning of verbal material (e.g., Dennis, Howard, and Howard, 2006; Weitz, O'Shea, Zook, and Needham, 2011). For example, a recent study by Weitz et al. (2011) showed correlation between the learning of a verbal sequence (Hebb digits task) and verbal working memory capacity. Note, however, that all of these latter findings regarding domain-specificity were related to explicit and not to implicit sequence learning. 
Stages of sequence learning - The differentiation between online and offline phases of learning also needs to be considered, as significant changes in the acquisition do not occur only during practice (online periods) but also between practice (offline) periods. The process that occurs during the offline periods is referred to as consolidation, which means stabilization of a memory trace after the initial acquisition; it can result in increased resistance to interference or even improvement in performance following an offline period (Krakauer and Shadmehr, 2006; Nemeth, Janacsek, Londe et al., 2010; Nemeth and Janacsek, 2011; Robertson, 2009; Song, 2009). The previously discussed studies measured sequence learning by one learning session without an offline period and barely showed working memory's effect on sequence learning. On the other hand, if we administer multiple learning sessions with, for example, 24-hour delay periods, we are able to examine the effect of consolidation processes on the relationship between sequence learning and working memory capacity. For example, Howard and Howard (1997) as well as Schwartz et al. (2003) administered more learning sessions distributed throughout several days and found significant working memory effects on a sequence learning task. However, they did not analyze the effect of consolidation specifically (the performance from all learning sessions were collapsed). Future studies need to test the relationship between sequence knowledge after a consolidation period and working memory capacity.

General skill vs. sequence-specific learning - There seem to be a number of misunderstandings regarding the sequence learning indices used in the studies focusing on the association between sequence learning and working memory. Recent studies highlight that at least two aspects of learning have to be differentiated in the sequence learning experiments. The RT performance improvement as a result of practice can be attributed both to general familiarization with the task (termed as general skill learning, or general practice effects) and to learning the sequential structure/regularity of the task specifically (termed as sequence- 
specific learning) (Janacsek and Nemeth, 2012; Song, Howard, and Howard, 2007). In the classical SRT task (Nissen and Bullemer, 1987), the more the participants practice, the faster they are on blocks containing the repeated sequential structure. When this sequence is changed to a random series of stimuli at the end of practice, participants' response rate becomes slower. In this task, sequence learning can be measured in different ways: 1) by the reaction time (RT) decrease in sequential blocks (i.e., participants are generally faster in the last sequence block compared to the first sequence block; e.g., Bo, Jennett, et al., 2011); 2) by the RT difference between the last sequence block and the subsequent random block. The latter measure is more widely accepted in sequence learning literature (e.g., Keele, Ivry, Mayr, Hazeltine, and Heuer, 2003; Robertson, 2007; for critical view see Reed and Johnson, 1994). For example, using these indices, Bo et al. (2011) found a positive correlation between working memory capacity and the rate of RT decrease (thus, the RT change in sequential blocks), but not between working memory and RT difference in the last sequence and the following random block (which is supposed to reflect sequence-specific learning better). In a more recent study, Bo, Jennett and Seidler (2012) replicated these results in elderly participants. One potential concern regarding these results is whether it is possible to separate the above mentioned general skill and sequence-specific learning components in the classical SRT task. Namely, the RT decrease in the sequential blocks can reflect both general skill and sequence-specific learning. The contribution of these two factors to performance improvement cannot be precisely determined. As Bo et al. $(2011,2012)$ found correlation only with the RT decrease in sequence blocks, not with the sequence/random difference score, we can suggest that working memory might be more related to general skill learning than to the sequence-specific learning. Therefore, further studies and different analysis methods are needed to clarify the relationship between working memory and general skill learning or sequence-specific learning. For example, as Verwey (1996) proposed, participants respond to 
individual sequence elements one by one at the beginning of the sequence learning, but consecutive elements can be formed into a single representation ("chunk") once the sequence is learned. Thus, it is possible to determine the mean chunk length in the SRT task with higher length (larger window into the sequence structure) reflecting better sequence-specific learning. Using this analysis method, Bo et al. (2009) found a relationship between working memory capacity and mean chunk length in explicit sequence learning. This raises the question of whether such a relationship is present between the mean chunk length in implicit sequence learning and working memory.

Another possible approach for future studies can be the use of probabilistic sequences instead of deterministic ones (as in the SRT task), since probabilistic second- or higher-order sequence regularities give us the opportunity to analyze sequence-specific and general skill learning separately and more precisely. For example, in the alternating SRT (Howard and Howard, 1997) task, repeating stimuli alternate with random ones, thus every second element in the stream is determined randomly. Hence, it is possible to track sequence-specific learning continuously by comparing responses to the random and sequence elements in all blocks. This could help to investigate the relationship between sequence-specific learning and working memory more precisely.

\section{Neurocognitive background of the relationship between working memory and sequence}

\section{learning}

A growing body of evidence suggests that the fronto-striatal circuit, including the caudate nucleus and lateral PFC, plays a critical role in working memory performance. In this circuit, PFC is thought to be responsible for the coordination of encoding, maintenance, and manipulation of information, by, for example, biasing the processing in posterior sensory-and multimodal association areas (Bar, 2003; Desimone and Duncan, 1995; Miller and Cohen, 
2001; Nobre, 2001). The striatum, on the other hand, modulates the working memory performance by increasing or decreasing the inhibition of the PFC (Ashby, Turner, and Horvitz, 2010). Recent studies highlight that the striatum is primarily involved in the manipulation processes, for example filtering out the irrelevant information (McNab and Klingberg, 2007), conflict monitoring (Beste et al., 2012), and sequencing (Riley, Moore, Cramer, and Lin, 2011).

In this fronto-striatal circuit, the last two decades of implicit sequence learning research showed the involvement of striatum in the acquisition of sequence knowledge (Keele et al., 2003; Rieckmann et al., 2010), while the role of PFC remained inconclusive. Determining the specific conditions where working memory capacity and sequence learning correlate can help us to unravel the complex role of PFC in cognition and specifically in sequence learning. In most studies finding correlation between these two measures, participants were aware of the sequence and had the intention to improve their performance utilizing this sequence knowledge. In these cases a higher extent of PFC-dependent coordination and cognitive control is implemented to perform the task. Supporting this argument, fMRI studies found greater PFC activation in this explicit/intentional version of sequence learning compared to the implicit/incidental one (e.g., Fletcher et al., 2005). Thus, the relationship between working memory capacity and sequence learning in these cases might be based on the mutual PFC-dependent coordination component of the performance.

However, in some cases implicit sequence learning was also correlated with working memory capacity. In most of these studies working memory capacity was measured by a complex task where the manipulation of the information, not only the maintenance, was relevant for a high task performance. Based on these results we can suggest that this observed correlation is primarily attributable to the greater involvement of the striatum in these working memory tasks. The recent studies showing the specific role of striatum in 
information manipulation are in line with this assumption (Beste et al., 2012; Riley et al., 2011). The other plausible explanation could be that most of the studies finding a relationship administered more sessions to measure sequence learning (Howard and Howard, 1997; Schwartz et al., 2003), allowing a better consolidation of the acquired information. One might assume that processes engaged in this offline phase of sequence learning share more similarity with working memory than the online sequence processing (e.g., maintaining the acquired information in an active state for a longer period can help stabilize the memory traces). However, these studies did not contrast the online and offline performance directly and did not involve brain imaging; therefore future research needs to clarify this issue.

\section{Summary}

In our review, we briefly touched on some relevant issues regarding the possible relationship between implicit sequence learning and working memory: 1) the explicitness of the sequence; 2) measures of working memory capacity; 3) online and offline stages of sequence learning; and 4) general skill- and sequence-specific learning. With these factors we can better interpret the results of studies on the relationship between sequence learning and working memory. However, note that because of the length limitation of the mini-review we could not critically investigate the question of whether the implicit sequence learning and working memory tasks discussed in this mini-review are the most adequate measures for tapping the constructs they were designed to tap (Kane, Conway, Miura, and Colflesh, 2007; Moisello et al., 2009; Unsworth and Engle, 2006).

Based on the studies included in this mini-review (Table 1), we suggest a relationship between working memory and 1) explicit rather than implicit sequence learning, 2) potentially to a higher extent with general skill learning than with sequence-specific learning, 3) with some specificity to verbal or visuospatial domains (i.e., higher correlation between 
visuospatial working memory and learning of visuospatial sequences than learning verbal ones). In the reviewed literature only two studies have administered multiple sessions to measure sequence learning. However, they analyzed the relationship between working memory and sequence learning by collapsing the online and offline components. Therefore, the effect of consolidation on this relationship remains an open question needing to be addressed in further research. In addition, future studies also would benefit from taking into account which measures are used for determining the working memory capacity (i.e., span or change detection tasks) as well as sequence learning (i.e., general RT improvements, RT difference between sequence and random elements, chunk length of the sequence, etc.).

Considering the factors discussed in this mini-review will aid in the design of future experiments, in the interpretation of results, and a deeper appreciation of the relationship between sequence learning and working memory and underlying brain structures.

Table 1. Studies investigating the relationship between sequence learning and working memory (WM). "Mixed" indicates when general skill and sequence-specific learning cannot be separated in the analysis method that the study used.

\begin{tabular}{|c|c|c|c|c|c|}
\hline Study & $\begin{array}{l}\text { Explicit } \\
\text { /Implicit }\end{array}$ & WM measure & $\begin{array}{l}\text { Online } \\
\text { /offline }\end{array}$ & $\begin{array}{c}\text { General skill } \\
\text { /sequence- } \\
\text { specific }\end{array}$ & WM effect \\
\hline \multirow{3}{*}{$\begin{array}{l}\text { Frensch and Miner } \\
\text { (1994), Exp. } 1\end{array}$} & Explicit & \multirow{3}{*}{ Span task } & \multirow{3}{*}{ Online } & \multirow{3}{*}{ Sequence-specific } & Yes \\
\hline & & & & & \\
\hline & Implicit & & & & No \\
\hline $\begin{array}{l}\text { Frensch and Miner } \\
\text { (1994), Exp. } 2\end{array}$ & Implicit & $\begin{array}{l}\text { Span task, dual } \\
\text { task condition }\end{array}$ & Online & Sequence-specific & Yes \\
\hline Feldman et al. (1995) & Implicit & Span task & Online & Sequence-specific & No \\
\hline \multirow{2}{*}{$\begin{array}{l}\text { Howard and Howard } \\
\text { (1997) }\end{array}$} & \multirow{2}{*}{ Implicit } & \multirow{2}{*}{ Span task } & $\begin{array}{c}\text { Online } \\
(\text { Session 1) }\end{array}$ & \multirow{2}{*}{ ificic } & $\begin{array}{c}\text { Not analyzed } \\
\text { separately }\end{array}$ \\
\hline & & & $\begin{array}{l}\text { Online + Offline } \\
(\text { Session 1-6) }\end{array}$ & & Yes \\
\hline
\end{tabular}




\begin{tabular}{|c|c|c|c|c|c|}
\hline \multirow{3}{*}{ Schwartz et al. (2003) } & \multirow{3}{*}{ Implicit } & \multirow{2}{*}{\multicolumn{2}{|c|}{$\begin{array}{c}\text { Online } \\
\text { (Session 1) }\end{array}$}} & \multirow{3}{*}{ Sequence-specific } & \multirow[t]{2}{*}{ No } \\
\hline & & & & & \\
\hline & & & $\begin{array}{l}\text { Online + Offline } \\
\text { (Session 1-6) }\end{array}$ & & Yes \\
\hline \multirow{6}{*}{$\begin{array}{l}\text { Unsworth and Engle } \\
\text { (2005) }\end{array}$} & & & & Sequence-specific & Yes \\
\hline & Explicit & Span task & Online & & \\
\hline & & & & Mixed & Yes \\
\hline & & & & Sequence-specific & No \\
\hline & Implicit & Span task & Online & & \\
\hline & & & & Mixed & No \\
\hline Bo et al. (2009) & Explicit & Change detection & Online & Sequence-specific & Yes \\
\hline \multirow[t]{4}{*}{ Kaufman et al. (2010) } & Implicit & Span task & Online & Sequence-specific & No \\
\hline & & & & Mixed & No \\
\hline & & Span task & & & \\
\hline & & & & Sequence-specific & No \\
\hline \multirow[t]{5}{*}{ Bo et al. (2011) } & Implicit & & Online & & \\
\hline & & & & Mixed & Yes \\
\hline & & Change detection & & & \\
\hline & & & & Sequence-specific & No \\
\hline & Explicit & & & & Yes \\
\hline \multirow[t]{3}{*}{ Weitz et al. (2011) } & & Span task & Online & Sequence-specific & \\
\hline & Implicit & & & & No \\
\hline & & & & Sequence-specific & No \\
\hline \multirow[t]{2}{*}{ Bo et al. (2012) } & Explicit & Change detection & Online & & \\
\hline & & & & Mixed & Yes \\
\hline
\end{tabular}

\section{Acknowledgements}

Thanks to our mentors Darlene V. Howard (Georgetown University), James H. Howard Jr. (The Catholic University of America) for helpful comments on the manuscript. Zsuzsa Londe (University of Southern California) and Zoltan Mari (Johns Hopkins University) helped us in the final version of the manuscript. Thanks to Clare Allyce Tucker for proof-reading and 
editing. This research was supported by Hungarian Science Foundation (OTKA-MB08 84743; OTKA K82068; OTKA NF 105878) and DAAD-MÖB (29775).

\section{References}

Ashby F G, Turner B O, and Horvitz J C. Cortical and basal ganglia contributions to habit learning and automaticity. Trends in Cognitive Sciences, 14 (5):208-215, 2010.

Bar M. A cortical mechanism for triggering top-down facilitation in visual object recognition. Journal of Cognitive Neuroscience, 15 (4):600-609, 2003.

Barnes K A, Howard J H Jr, Howard D V, Gilotty L, Kenworthy L, Gaillard W D, and _Vaidya C D. Intact implicit learning of spatial context and temporal sequences in childhood autism spectrum disorder. Neuropsychology, 22 (5):563-570. 2008.

Beste C, Ness V, Lukas C, Hoffmann R, Stüwe S, Falkenstein M, and Saft C. Mechanisms mediating parallel action monitoring in fronto-striatal circuits. Neuroimage, 62 (1):137-146, 2012.

Bo J, Borza V, and Seidler R D. Age-related declines in visuospatial working memory correlate with deficits in explicit motor sequence learning. Journal of Neurophysiology, 102 (5):2744-2754, 2009.

Bo J, Jennett S, and Seidler R. Working memory capacity correlates with implicit serial reaction time task performance. Experimental Brain Research, 214 (1):73-81, 2011.

Bo J, Jennett S, and Seidler R. Differential working memory correlates for implicit sequence performance in young and older adults. Experimental brain research, 221 (4):467477, 2012.

Bo J, Peltier S, Noll D, and Seidler R. Symbolic representations in motor sequence learning. Neuroimage, 54 (1):417-426, 2011. 
Brown J, Aczel B, Jimenez L, Kaufman S B, and Grant K P. Intact implicit learning in autism spectrum conditions. The Quarterly Journal of Experimental Psychology, 63 (9):1789$1812,2010$.

Brown R M, and Robertson E M. Inducing motor skill improvements with a declarative task. Nature Neuroscience, 10 (2):148-149, 2007.

Champod A S, and Petrides M. Dissociation within the frontoparietal network in verbal working memory: a parametric functional magnetic resonance imaging study. The Journal of Neuroscience, 30 (10):3849-3856, 2010.

Cowan N. Attention and Memory: An Integrated Framework. Oxford: Oxford University Press, 1995.

Dennis N A, Howard J H Jr, and Howard D V. Implicit sequence learning without motor sequencing in young and old adults. Experimental Brain Research, 175 (1):153-164, 2006.

Desimone R, and Duncan J. Neural mechanisms of selective visual attention. Annual review of neuroscience, 18 (1):193-222, 1995.

Destrebecqz A, Peigneux P, Laureys S, Degueldre C, Del Fiore G, Aerts J, Luxen A, Van der Linden M, Cleeremans A, and Maquet P. The neural correlates of implicit and explicit sequence learning: Interacting networks revealed by the process dissociation procedure. Learning \& Memory, 12 (5):480-490, 2005.

Feldman J, Kerr B, and Streissguth A P. Correlational analyses of procedural and declarative learning performance. Intelligence, 20 (1):87-114, 1995.

Fletcher P C, Zafiris O, Frith C D, Honey R A E, Corlett P R, Zilles K, and Fink G R. On the benefits of not trying: brain activity and connectivity reflecting the interactions of explicit and implicit sequence learning. Cerebral Cortex, 15 (7):1002-1015, 2005. 
Foerde K, Knowlton B J, and Poldrack R A. Modulation of competing memory systems by distraction. Proceedings of the National Academy of Science, 103 (31):11778-11783, 2006.

Frank M J, O'Reilly R C, and Curran T. When Memory Fails, Intuition Reigns: Midazolam Enhances Implicit Inference in Humans. Psychological Science, 17 (8):700-707, 2006.

Frensch P A, and Miner C S. Effects of presentation rate and individual differences in shortterm memory capacity on an indirect measure of serial learning. Memory and Cognition, 22 (1):95-110, 1994.

Honda M, Deiber M P, Ibanez V, Pascual-Leone A, Zhuang P, and Hallett M. Dynamic cortical involvement in implicit and explicit motor sequence learning. A PET study. Brain, 121 (11):2159-2173, 1998.

Howard J H Jr, and Howard D V. Age differences in implicit learning of higher-order dependencies in serial patterns. Psychology and Aging, 12 (4):634-656, 1997.

Janacsek K, and Nemeth D. Predicting the future: From implicit learning to consolidation. International Journal of Psychophysiology, 83 (2):213-221, 2012.

Jiménez L. Attention and implicit learning. _Amsterdam/Philadelphia: John Benjamins Publishing Company, 2003.

Kane M J, Conway A R A, Miura T K, and Colflesh G J H. Working memory, attention control, and the N-back task: a question of construct validity. Journal of experimental psychology: learning, memory, and cognition, 33 (3):615-622, 2007.

Kaufman S B, DeYoung C G, Gray J R, Jiménez L, Brown J, and Mackintosh N. Implicit learning as an ability. Cognition, 116(3):321-340, 2010.

Keele S W, Ivry R, Mayr U, Hazeltine E, and Heuer H. The cognitive and neural architecture of sequence representation. Psychological Review, 110 (2):316-339, 2003. 
Krakauer J W, and Shadmehr R. Consolidation of motor memory. Trends in Neurosciences, 29 (1):58-64, 2006.

McNab F, and Klingberg T. Prefrontal cortex and basal ganglia control access to working memory. Nature Neuroscience, 11 (1):103-107, 2007.

Miller E K, and Cohen J D. An integrative theory of prefrontal cortex function. Annual review of neuroscience, 24 (1):167-202, 2001.

Moisello C, Crupi D, Tunik E, Quartarone A, Bove M, Tononi G, and Ghiraldi M F. The serial reaction time task revisited: a study on motor sequence learning with an armreaching task. Experimental brain research, 194(1):143-155, 2009.

Nemeth D, Csábi E, Janacsek K, Varszegi M, and Mari Z. Intact implicit probabilistic sequence learning in obstructive sleep apnea. Journal of Sleep Research, 21 (4):396401, 2012.

Nemeth D, Janacsek K, Balogh V, Londe Z, Mingesz R, Fazekas M, Jambori S, Danyi I, and Vetro A. Learning in Autism: Implicitly Superb. PloS ONE, 5 (7):e11731, 2010.

Nemeth D, Janacsek K, Londe Z, Ullman M T, Howard D, and Howard J H Jr. Sleep has no critical role in implicit motor sequence learning in young and old adults. Experimental Brain Research, 201 (2):351-358, 2010.

Nemeth D, and Janacsek K. The Dynamics of Implicit Skill Consolidation in Young and Elderly Adults. Journal of Gerontology: Psychological Sciences, 66B (1):15-22, 2011.

Nemeth D, Janacsek K, Polner B, and Kovacs Z A. Boosting Human Learning by Hypnosis. Cerebral Cortex, DOI:10.1093/cercor/bhs068, in press.

Nissen M J, and Bullemer P. Attentional requirements of learning: Evidence from performance measures. Cognitive Psychology, 19 (1):1-32, 1987. 
Nobre A C. The attentive homunculus: now you see it, now you don't. Neuroscience and biobehavioral reviews, 25 (6):477-496, 2001.

Pascual-Leone A, Wassermann E M, Grafman J, and Hallett M. The role of the dorsolateral prefrontal cortex in implicit procedural learning. Experimental Brain Research, 107 (3):479-485, 1996.

Poldrack R A, Clark J, Pare-Blagoev E J, Shohamy D, Creso Moyano J, Myers C, and Gluck M A. Interactive memory systems in the human brain. Nature, 414 (6863):546-550, 2001.

Reed J, and Johnson P. Assessing implicit learning with indirect tests: determining what is learned about sequence structure. Journal of Experimental Psychology: Learning, Memory, and Cognition, 20 (3):585-594, 1994.

Rieckmann A, Fischer H, and Bäckman L. Activation in striatum and medial temporal lobe during sequence learning in younger and older adults: Relations to performance. Neuroimage, 50 (3):1303-1312, 2010.

Riley J D, Moore S, Cramer S C, and Lin J J. Caudate atrophy and impaired frontostriatal connections are linked to executive dysfunction in temporal lobe epilepsy. Epilepsy \& Behavior, 21 (1):80-87, 2011.

Robertson E M. The Serial Reaction Time Task: Implicit Motor Skill Learning? Journal of Neuroscience, 27 (38):10073-10075, 2007.

Robertson E M. From creation to consolidation: A novel framework for memory processing. PLoS Biology, 7 (1):e1000019, 2009.

Robertson E M, Tormos J M, Maeda F, and Pascual-Leone A. The role of the dorsolateral prefrontal cortex during sequence learning is specific for spatial information. Cerebral Cortex, 11 (7):628-635, 2001. 
Schwarb H, and Schumacher E H. Neural evidence of a role for spatial response selection in the learning of spatial sequences. Brain Research, 1247:114-125, 2009.

Schwartz B L, Howard D V, Howard J H Jr, and Hovaguimian A. Implicit Learning of Visuospatial Sequences in Schizophrenia. Neuropsychology, 17 (3):517-533, 2003.

Sefcsik T, Nemeth D, Janacsek K, Hoffmann I, Scialabba J, Klivenyi P, Ambrus G G, Haden $\mathrm{G}$, and Vecsei L. The role of the putamen in cognitive functions-A case study. Learning \& Perception, 1 (2):215-227, 2009.

Smith E E, and Jonides J. Storage and executive processes in the frontal lobes. Science, 283 (5408):1657-1661, 1999.

Song S. Consciousness and the consolidation of motor learning. Behavioural Brain Research, 196 (2):180-186, 2009.

Song S, Howard J H Jr, and Howard D V. Sleep does not benefit probabilistic motor sequence learning. Journal of Neuroscience, 27 (46):12475-12483, 2007.

Unsworth N, and Engle R W. Individual differences in working memory capacity and learning: Evidence from the serial reaction time task. Memory \& cognition, 33 (2):213-220, 2005.

Unsworth N, and Engle R W. Simple and complex memory spans and their relation to fluid abilities: Evidence from list-length effects. Journal of Memory and Language, 54 (1):68-80, 2006.

Verwey W B. Buffer loading and chunking in sequential keypressing. Journal of Experimental Psychology: Human Perception and Performance, 22 (3):544-562, 1996.

Vicari S, Verucci L, and Carlesimo G A. Implicit memory is independent from IQ and age but not from etiology: Evidence from Down and Williams syndromes. Journal of Intellectual Disability Research, 51 (12):932-941, 2007. 
Weitz D, O'Shea G, Zook N, and Needham W. Working memory and sequence learning in the Hebb digits task: Awareness is predicted by individual differences in operation span. The American journal of psychology, 124 (1):49-62, 2011. 